\title{
Why do results conflict regarding the prognostic value of the methylation status in colon cancers? the role of the preservation method
}

Benjamin Tournier ${ }^{1,2^{*}}$, Caroline Chapusot ${ }^{1,2,3}$, Emilie Courcet ${ }^{1,2}$, Laurent Martin ${ }^{2,4}$, Côme Lepage ${ }^{1,3}$, Jean Faivre L $^{1,3}$ and Françoise Piard ${ }^{1,2,3,4}$

\begin{abstract}
Background: In colorectal carcinoma, extensive gene promoter hypermethylation is called the CpG island methylator phenotype (CIMP). Explaining why studies on CIMP and survival yield conflicting results is essential. Most experiments to measure DNA methylation rely on the sodium bisulfite conversion of unmethylated cytosines into uracils. No study has evaluated the performance of bisulfite conversion and methylation levels from matched cryo-preserved and Formalin-Fixed Paraffin Embedded (FFPE) samples using pyrosequencing.
\end{abstract}

Methods: Couples of matched cryo-preserved and FFPE samples from 40 colon adenocarcinomas were analyzed. Rates of bisulfite conversion and levels of methylation of LINE-1, MLH1 and MGMT markers were measured.

Results: For the reproducibility of bisulfite conversion, the mean of bisulfite-to-bisulfite standard deviation (SD) was $1.3 \%$. The mean of run-to-run SD of PCR/pyrosequencing was $0.9 \%$. Of the 40 DNA couples, only $67.5 \%, 55.0 \%$, and $57.5 \%$ of FFPE DNA were interpretable for LINE-1, MLH1, and MGMT markers, respectively, after the first analysis. On frozen samples the proportion of well converted samples was $95.0 \%, 97.4 \%$ and $87.2 \%$ respectively. For DNA showing a total bisulfite conversion, 8 couples (27.6\%) for LINE-1, 4 couples (15.4\%) for MLH1 and 8 couples (25.8\%) for MGMT displayed significant differences in methylation levels.

Conclusions: Frozen samples gave reproducible results for bisulfite conversion and reliable methylation levels. FFPE samples gave unsatisfactory and non reproducible bisulfite conversions leading to random results for methylation levels. The use of FFPE collections to assess DNA methylation by bisulfite methods must not be recommended. This can partly explain the conflicting results on the prognosis of CIMP colon cancers.

\section{Background}

Epigenetic dysregulation is a major event in the origin of many cancers [1]. DNA methylation, the most widely studied epigenetic mechanism, occurs in cytosines that precede guanines ( $\mathrm{CpG}$ dinucleotides). The $\mathrm{CpG}$ dinucleotides may be found concentrated in regions called $\mathrm{CpG}$ islands, commonly located in gene promoters. In colon cancers, a number of tumour suppressor genes are transcriptionally silenced by promoter $\mathrm{CpG}$ island hypermethylation $[2,3]$. Among them, one subset referred to as the $\mathrm{CpG}$ island methylator phenotype

\footnotetext{
* Correspondence: benjamin.tournier@chu-dijon.fr 'Institut National de la Sante et de la Recherche Médicale, Université de Bourgogne, U866 Dijon, France

Full list of author information is available at the end of the article
}

(CIMP) exhibits widespread promoter methylation $[2,4]$. Studies on CIMP status and survival in colon cancers have yielded somewhat inconsistent results [5-13]. One of our previous studies [5] as well as other studies $[8,11,13]$ suggested that the CIMP had an adverse effect on survival in MSS (Microsatellite Stable) tumours, while in other reports CIMP-H (CIMP-High) status was independently associated with low specific mortality $[9,12]$. These discrepancies might result from differences in the choice of tissue samples. The quality of DNA samples depends especially on the material available which may be cryo-preserved or formalin-fixed paraffin embedded (FFPE) tissue. The cryo-preservation technique provides the best protection of the DNA, but these specimens are far less common than FFPE tissue from

\section{Biomed Central}

(c) 2011 Tournier et al; licensee BioMed Central Ltd. This is an Open Access article distributed under the terms of the Creative Commons Attribution License (http://creativecommons.org/licenses/by/2.0), which permits unrestricted use, distribution, and reproduction in any medium, provided the original work is properly cited. 
pathology departments, which is a vast resource. The quality of archived specimens (FFPE tissues) depends on the fixation and storage conditions employed and can vary greatly from one sample to another. Moreover, formalin fixation induces degradation and cross-linkings between proteins and proteins/DNA bases. FFPE tissues thus produce a poorer yield of DNA. To evaluate DNA methylation, the gold-standard method is based on sodium bisulfite conversion [14], in which unmethylated cytosines are converted into uracils. Then, after PCR, it is possible to differentiate unmethylated cytosines (replaced by thymines) from methylated cytosines which are protected from bisulfite conversion [15]. However, the DNA bisulfite conversion step is a chemical reaction that also degrades DNA. All things considered, crosslinkings and DNA degradation caused by fixation, extraction and conversion methods have a negative impact on conversion efficacy, while DNA conversion must be total and reproducible to allow meaningful interpretation of results.

A number of techniques have been employed to analyze converted DNA [16-19]. These include MSP (methylation-specific polymerase chain reaction), Methylight (real time PCR), SMART-MSP (Sensitive Melting Analysis after Real Time PCR - methylation-specific polymerase chain reaction), MS-HRM (Methylation Sensitive - High Resolution Melting) and pyrosequencing. All these techniques are based on DNA bisulfite conversion. Among them, pyrosequencing is the only one that comprises an in-built measure to check the completeness of bisulfite conversion (conversion control) which allows a precise evaluation of the quality of the conversion. Moreover, it gives the percentage of methylated allele for each CpG dinucleotide analysed.

It is important to investigate why there are discrepancies in the prognostic value of the CIMP phenotype in colorectal cancers between studies especially as few explanations have been proposed. The aim of this study was to evaluate the feasibility of analyzing DNA methylation from DNA extracted from FFPE tissues and to compare the results with those obtained from frozen material using pyrosequencing technology. To this end, three different known markers of methylation (LINE-1, MLH1 and MGMT) were chosen. Results of this work could help establish a standard method for assessing DNA methylation and thus make it possible to compare results obtained in this field.

\section{Methods}

\section{Samples}

Forty tumour tissue samples from patients resected for a colon adenocarcinoma were included. For each tumour, one frozen sample previously stored in the Ferdinand Cabanne Biological Resources Centre (Dijon, France) and one FFPE (Formalin Fixed Paraffin Embedded) tissue block, were available. The CPP EST I committee (Comité de Protection des Personnes: Protection of Individuals committee) approved the use of these biological collections. The tissue samples were considered surgical waste in accordance with French ethical laws (L.1211-3 to L.1211-9).

Quality control of frozen samples was carried out before DNA extraction according to a strict process. Three sections, each separated by 15 other sections, were cut on a cryostat and stained with HemateinEosin-Safran (HES). These three stained sections were analyzed by a pathologist and the proportion of tumour tissue was recorded. The sections retained for the study were the consecutive sections located between two stained sections containing at least $40 \%$ of tumour cells. The choice of the percentage of tumor cellularity was established in our laboratory from previous analyses (data not shown). This percentage was ample for methylation quantification.

For FFPE samples, the slides were also reviewed by a pathologist in order to select an area rich in tumour cells (> 40\% of tumour cells). The initial paraffin blocks were manually dissected and the selected tumour area was embedded in a new paraffin block.

For all pairs of FFPE/frozen samples, we always checked that the difference in tumour cellularity between selected sections was slight in order to be able to compare both tissues.

\section{DNA extraction from frozen tissues: Nucleospin ${ }^{\circledR} 96$ tissue kit (Macherey Nagel ${ }^{\circledR}$ )}

Twenty $50 \mu \mathrm{m}$-tissue sections were crushed with two stainless steel balls in a mixture containing RLT buffer (Qiagen ${ }^{\circledR}$ ) and 1\% $\beta$-mercapto-ethanol (14.3 M; Sigma Aldrich $\left.^{(}\right)$. Half of each sample was then centrifuged for $10 \mathrm{~min}$ (10000 rcf (relative centrifugal force)). The pellets were suspended in $180 \mu \mathrm{l}$ of lysis buffer (T1 buffer) and $25 \mu$ l proteinase K. Samples were kept at $56^{\circ} \mathrm{C}$ overnight. DNA extraction was performed with a TECAN ${ }^{\circledR}$ automate following the supplier's recommendations. DNA was released in $100 \mu$ l elution buffer.

\section{DNA extraction from formalin-fixed paraffin embedded (FFPE) tissues \\ Classical method}

For each sample, ten $15 \mu \mathrm{m}$-thick tissue sections underwent proteinase $\mathrm{K}$ digestion (> $600 \mathrm{mAU} / \mathrm{ml}$, Qiagen ${ }^{\circledR}$ ) at $56^{\circ} \mathrm{C}$ for one night. DNA was extracted using a Bionobis kit (Magtration ${ }^{\circledR}-$ Magazorb $^{\circledR}$ ) and a Bionobis ${ }^{\circledR}$ automat according to the manufacturer's instructions. The samples were lysed, washed and then adsorbed onto magnetic silicate particles. DNA was immobilized by magnetic attraction, washed again and released in 
$100 \mu \mathrm{l}$ of water. DNA extracted with this technique was analyzed for the LINE-1 marker.

\section{Dedicated Method to FFPE tissue: QIAamp ${ }^{\circledR}$ DNA FFPE Tissue kit (QIAGEN ${ }^{\circledR}$ )}

For each sample, ten $15-\mu$ m-thick tissue sections were transferred into a $1.5 \mathrm{ml}$ tube. The extraction was performed according to the manufacturer's protocol. For further details see Additional file 1. In brief, tissue sections were first dewaxed using toluene and lysed under denaturing conditions with proteinase $\mathrm{K}$. Then lysates were incubated at $90^{\circ} \mathrm{C}$. DNA was bound to the membrane and contaminants were washed away by several washing steps. Finally pure DNA was eluted.

\section{Quantification and quality assessment of DNA}

DNA was quantified with a Nanodrop ${ }^{\circledR}$ spectrophotometer (Thermo scientific ${ }^{\circledR}$ ) and diluted at $50 \mathrm{ng} / \mu \mathrm{l}$. The quality of the DNA was assessed by multiplex PCR which amplified microsatellite regions as previously described $[20,21]$.

DNA bisulfite conversion - EpiTect Bisulfite kit (QIAGEN ${ }^{\circledR}$ ) For bisulfite conversion, the optimal quantity of DNA was determined in a preliminary work (data not shown). Five hundred nanograms of genomic DNA of each sample were used and bisulfite treatment was performed according to the manufacturer's protocol. For further details see Additional file 1. In brief, first the bisulfite mediated conversion of unmethylated cytosines was performed. After, the converted single strand DNA was bound to the membrane of the EpiTect spin columns. Membrane bound DNA was washed, then desulfonated, and washed again to remove the desulfonation agent. Finally pure converted DNA was eluted.

\section{PCR and pyrosequencing assays}

We previously optimized PCR conditions for each amplification by testing the following conditions: annealing temperature, magnesium concentration and cycle number.

\section{Measurement of LINE-1 methylation level}

LINE-1 PCR amplifies a 154 base-pair sequence in the consensus promoter of LINE-1 elements (acc. $\mathrm{n}^{\circ}$ $\mathrm{X} 58075$ ). It was performed using custom primers (Additional file 2), designed with the PyroMark assay design 2.0 (Qiagen $^{\circledR}$ ). The PCR reaction was carried out in a 50 $\mu \mathrm{L}$ final volume comprising $25 \mu \mathrm{L}$ of PyroMark Master Mix (containing PCR buffer, dNTP and HotStar Taq DNA polymerase), $5 \mu \mathrm{L}$ of Coral Load buffer, $4 \mu \mathrm{L}$ of $25 \mathrm{mM} \mathrm{MgCl}_{2}, 1 \mu \mathrm{L}$ of the forward and biotinylated reverse primers $(0.2 \mu \mathrm{M}$ final concentration), $13 \mu \mathrm{L}$ of RNase free water and $1 \mu \mathrm{L}$ of bisulfite treated DNA (Qiagen ${ }^{\circledR}$ ). PCR cycling conditions were as follows: initial denaturing at $95^{\circ} \mathrm{C}$ for $15 \mathrm{~min}, 45$ cycles of $95^{\circ} \mathrm{C}$ for $30 \mathrm{~s}, 56^{\circ} \mathrm{C}$ for $30 \mathrm{~s}$, and $72^{\circ} \mathrm{C}$ for $30 \mathrm{~s}$ and final extension at $72^{\circ} \mathrm{C}$ for $10 \mathrm{~min}$. Reverse single-stranded biotinylated templates were isolated using the PyroMark Vacuum Prep WorkStation (Qiagen $\left.{ }^{(}\right)$). Forty-six microlitres of PCR product were added to $38 \mu \mathrm{L}$ of binding buffer (Qiagen ${ }^{\circledR}$ ) and $2 \mu \mathrm{L}$ streptavidin sepharose highperformance beads (GE Healthcare $\left.{ }^{(}\right)$. The mixtures were shaken for $10 \mathrm{~min}$ at $1400 \mathrm{rpm}$ (revolution per minute). After agitation, beads covered by biotinylated DNA were collected and retained on filter probes by permanent vacuum. The filter probes were successively immerged in different baths: in ethanol $70 \%$ for $5 \mathrm{~s}$, in PyroMark denaturation solution for $5 \mathrm{~s}$ and in PyroMark wash buffer $1 \times$ for $15 \mathrm{~s}$ (Qiagen ${ }^{\circledR}$ ). Then the vacuum was turned off and the beads fixing DNA strands were released into a 24 well plate containing 25 $\mu \mathrm{l}$ of annealing buffer with $0.3 \mu \mathrm{M}$ of sequencing primer in each well. The sequencing plate was kept at $80^{\circ} \mathrm{C}$ for $2 \mathrm{~min}$ and at room temperature for $5 \mathrm{~min}$. Pyrosequencing reactions were performed in a PyroMark Q24 MDx system using PyroGold reagents $\left(\right.$ Qiagen $^{\circledR}$ ). The nucleotide dispensation order used is indicated in Additional file 2. Results were analyzed using PyroMark Q24 2.0.6 Software. To ensure successful bisulfite conversion of unmethylated cytosines, an internal conversion control that corresponded to the position of a non-CG cytosine (not subject to methylation) was present in the dispensation sequence. The average LINE-1 methylation level was calculated as the mean of the proportions of $C$ (\%) at the $3 \mathrm{CpG}$ sites analysed, which were located at positions $+319,+322$ and +329 (positions of the corresponding Guanine in the forward DNA strand, in relation to the first nucleotide base of the consensus promoter sequence) and this indicated the level of methylation of $L I N E-1$ elements.

\section{Measurement of MLH1 and MGMT methylation levels}

To investigate the methylation level of $M L H 1$ and MGMT genes, we used the PyroMark Q24 kits (Qiagen $\left.{ }^{\circledR}\right)$ as we did not succeed in designing amplicons targeting the same CpG sites with a smaller length than those proposed in these kits. The MLH1 kit was designed to detect the methylation level in a region -209 to -181 from the transcription start site of the $M L H 1$ gene and the MGMT kit to detect the methylation level in a region +17 to +39 in exon 1 of the MGMT gene. PCR reactions were performed according to the manufacturer's instructions. Then, $22 \mu \mathrm{L}$ of PCR product was added to $40 \mu \mathrm{L}$ of binding buffer, $16 \mu \mathrm{L}$ of ultrapure water and $2 \mu \mathrm{L}$ of streptavidin sepharose high-performance beads. The single-stranded biotinylated templates were purified similarly to the LINE-1 assay (mentioned above). The sequencing plate containing purified DNA strands and sequencing primer was kept at $80^{\circ} \mathrm{C}$ for 2 min and at room temperature for $5 \mathrm{~min}$. 
Pyrosequencing reactions were performed in a PyroMark Q24 MDx system using PyroGold reagents (Qiagen $\left.^{(}\right)$. The nucleotide dispensation orders of $M L H 1$ and MGMT assays are indicated in Additional file 2. Results were analyzed using PyroMark Q24 2.0.6 Software. To ensure successful bisulfite conversion of unmethylated cytosines, an internal conversion control that corresponded to the position of a non-CG cytosine (not subject to methylation) was present in the dispensation sequence. The average methylation level for the two markers was calculated as the mean of the proportions of $\mathrm{C}(\%)$ at the $5 \mathrm{CpG}$ sites that were analysed.

\section{Assessment of reproducibility of bisulfite conversion, PCR/Pyrosequencing}

The reproducibility of bisulfite conversion efficiency and PCR/Pyrosequencing and the variability of methylation measurement generated using the same frozen DNA sample were evaluated as shown in Figure 1. In practice, a pool of tumour DNA from cryo-preserved tissues was used; identical bisulfite conversions were performed one day apart (day 1: conversion A, day 2: conversion $\mathrm{B}$ ). Four conversions were carried out on day 1 (A1, A2, A3, A4) and two on day 2 (B1 and B2). Then, two independent PCRs (one day apart) were carried out in duplicate from each converted sample and then two independent pyrosequencing procedures, one day apart, were also performed. We measured the level of LINE-1 methylation for the three sites by pyrosequencing on each of these 24 PCR products [six converted DNA samples $\times$ two $\mathrm{PCR} \times 2$ (in duplicate)]. For each case and each site we measured the standard deviations (SD) on A1 through B2 of the four measures of levels of methylation, which could primarily depend on variations in bisulfite conversion. The repetition of PCR and Pyrosequencing allowed us to determine the exact levels of methylation on the set of bisulfite-converted DNA samples. The SD calculated between the different measures of methylation of each site for each given converted sample could primarily depend on day-to-day variations in the pyrosequencing assay. In addition, the maximum variation in the methylation level in absolute value was calculated for each site.

\section{Results}

Variability of measurement of the methylation level of DNA from cryo-preserved tissue

Data for LINE-1 methylation levels of the pool of tumour DNA sample for the three CpG sites analyzed are summarized in Table 1. The mean of SDs observed after bisulfite treatments (bisulfite-to-bisulfite SD) was $1.3 \%$ and the mean of SDs observed after PCR/pyrosequencing was $0.9 \%$. The variability of measurement of the methylation level induced by bisulfite conversion

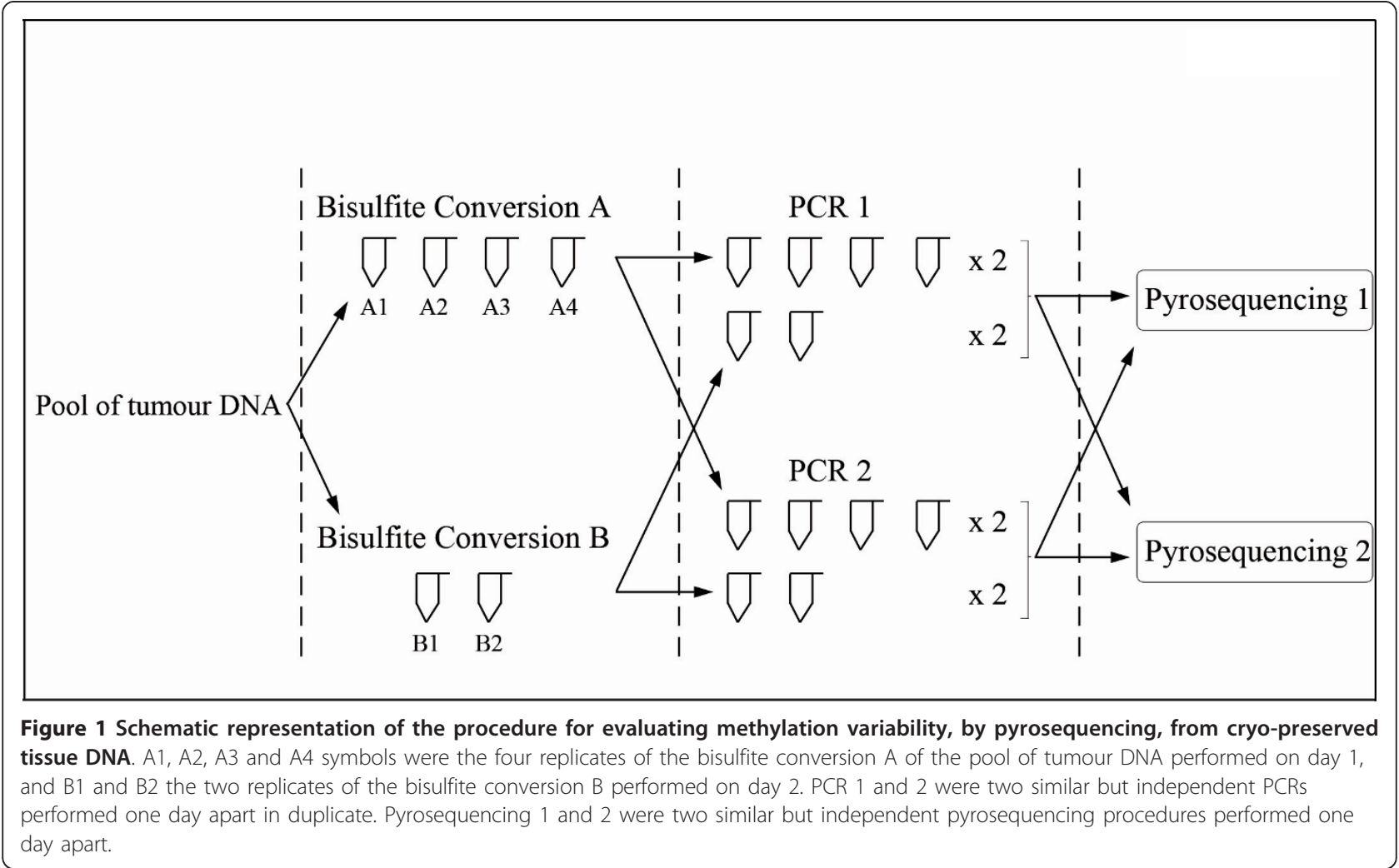


Table 1 Variability of LINE-1 methylation levels of DNA from cryo-preserved tissue

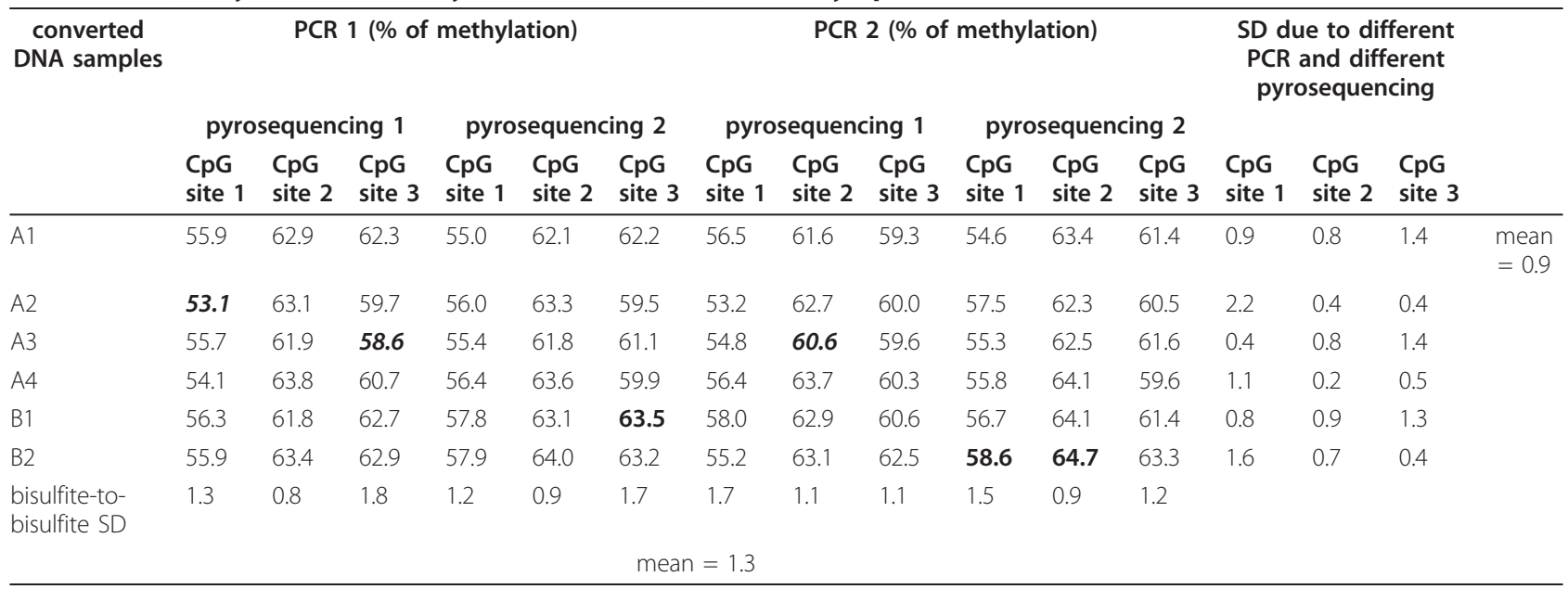

\begin{tabular}{lll}
\hline SD between all measures of site 1 & $1.4 \%$ \\
\hline SD between all measures of site 2 & $1.0 \%$ \\
\hline SD between all measures of site 3 & $1.4 \%$ \\
\hline
\end{tabular}

The levels of methylation (\%) of the three studied CpG sites are given according to the converted DNA sample analysed (A1 through B2) and for each condition of analysis (PCR and pyrosequencing). Numbers in bold italics indicate minimal values of methylation on all measures for each $\mathrm{CpG}$ site. Numbers in bold indicate maximal values of methylation on all measures for each $\mathrm{CpG}$ site

and PCR/pyrosequencing was $1.4 \%$. for site $1,1.0 \%$ for site 2 and $1.4 \%$ for site 3 .

\section{Qualitative analysis of bisulfite conversion for FFPE tissues \\ Classical extraction method}

For DNA extracted with the classical method, the bisulfite conversion was tested for the $L I N E-1$ marker. Twenty five DNA samples displayed an uncertain ("check": 5.0 to $7.0 \%$ of unconverted cytosines) or poor conversion ("failed": more than $7.0 \%$ of unconverted cytosines) in the first analysis and 24 in the second analysis. The results of the control of conversion were not always similar in the two analyses. Cases $n^{\circ} 2,5,6,9$, 14, 27 and 37 showed invalid control of conversion ("check" or "failed") during the first analysis and valid control ("passed": less than $5.0 \%$ of unconverted cytosines) of conversion during the second analysis. On the other hand, six cases $\left(n^{\circ} 8,18,20,22,23\right)$ showed valid conversion after the first assay and invalid control of conversion at the second analysis. For nine cases $\left(\mathrm{n}^{\circ} 3\right.$, $16,24,28,30,32,33,36,38)$ the controls showed valid conversion for the two analyses. By combining the valid results of the two analyses, $55.0 \%$ of the cases (22 cases) were interpretable.

\section{Dedicated extraction kit for the LINE-1 marker}

All the DNAs could be amplified. The internal controls showed valid conversion for 27 cases (67.5\%) in the first analysis and 28 cases (70.0\%) after the second analysis with the LINE-1 marker. Cases ${ }^{\circ}$ 2, 11 and 19 showed invalid control of conversion ("check" or "failed") in the first analysis and valid control of conversion ("passed") in the second analysis. On the other hand, two cases $\left(\mathrm{n}^{\circ}\right.$ 4,39 ) showed successful conversion after the first assay and an invalid control of conversion in the second analysis. By combining the valid results of the two analyses, $75.0 \%$ of the cases were interpretable (30 of the $40 \mathrm{FFPE}$ DNAs).

\section{Dedicated extraction kit for MLH1 and MGMT}

With the $M L H 1$ marker, 11 (27.5\%) DNAs could not be amplified. Among those that were amplified (29/40), controls showed valid conversion for 23 cases $(57.5 \%$ of the 40 cases) in the first assay and 27 after combining the two analyses. Concerning the MGMT marker, all the DNAs could be amplified. The internal controls of conversion were valid for 22 cases $(55.0 \%)$ in the first analysis. Among the 18 cases which gave an invalid control of conversion, $13\left(\mathrm{n}^{\circ} 4,7,17,19,20,22,26,30,33\right.$, $34,35,39$ and 40) gave a valid control of conversion in the second analysis. By combining the valid results, 35 of the 40 (87.5\%) FFPE DNAs showed valid control of conversion.

\section{Qualitative analysis of bisulfite conversion for cryo- preserved tissues}

The results of bisulfite conversion for the three markers after two analyses are given in Table 2. Assessment of the LINE-1 marker showed that two frozen samples failed to be converted (cases 19 and 27) in the first assay (95.0\% of well converted DNA) and one was not amplified after the two analyses (case 27). For the MLH1 marker, in the first assay, only one DNA ( $\left.\mathrm{n}^{\circ} 21\right)$ 
Table 2 Results of internal controls of conversion of the three markers

\begin{tabular}{|c|c|c|c|c|c|c|c|c|c|c|c|c|c|c|}
\hline \multirow[t]{3}{*}{ Identity } & \multirow{2}{*}{\multicolumn{2}{|c|}{$\begin{array}{c}\text { FFPE DNA } 1 \\
\text { LINE-1 }\end{array}$}} & \multirow{2}{*}{\multicolumn{2}{|c|}{$\begin{array}{c}\text { FFPE DNA } 2 \\
\text { LINE-1 }\end{array}$}} & \multirow{2}{*}{\multicolumn{2}{|c|}{$\begin{array}{c}\text { FFPE DNA } 2 \\
M L H 1\end{array}$}} & \multirow{2}{*}{\multicolumn{2}{|c|}{$\begin{array}{c}\text { FFPE DNA } 2 \\
M G M T\end{array}$}} & \multirow{2}{*}{\multicolumn{2}{|c|}{$\begin{array}{c}\text { Cryo. DNA } \\
\text { LINE-1 }\end{array}$}} & \multirow{2}{*}{\multicolumn{2}{|c|}{$\begin{array}{c}\text { Cryo. DNA } \\
M L H 1\end{array}$}} & \multirow{2}{*}{\multicolumn{2}{|c|}{$\begin{array}{c}\text { Cryo. DNA } \\
M G M T\end{array}$}} \\
\hline & & & & & & & & & & & & & & \\
\hline & PCR 1 & PCR 2 & PCR 1 & PCR 2 & PCR 1 & PCR 2 & PCR 1 & PCR 2 & PCR 1 & PCR 2 & PCR 1 & PCR 2 & PCR 1 & PCR 2 \\
\hline 1 & check & failed & passed & passed & passed & & passed & & passed & passed & passed & & passed & \\
\hline 2 & failed & passed & check & passed & 1 & & passed & & passed & passed & passed & & passed & \\
\hline 3 & passed & passed & check & check & 1 & & failed & failed & passed & passed & passed & & passed & \\
\hline 4 & failed & failed & passed & check & 1 & & failed & passed & passed & passed & passed & & passed & \\
\hline 5 & failed & passed & failed & check & 1 & passed & passed & passed & passed & passed & passed & & passed & \\
\hline 6 & check & passed & passed & passed & passed & & passed & & passed & passed & passed & & passed & \\
\hline 7 & failed & failed & failed & check & passed & passed & failed & passed & passed & passed & passed & & check & check \\
\hline 8 & passed & failed & passed & passed & passed & & passed & & passed & passed & passed & & passed & \\
\hline 9 & check & passed & passed & passed & passed & & passed & & passed & passed & passed & & passed & \\
\hline 10 & failed & failed & passed & passed & passed & & passed & & passed & passed & passed & & passed & \\
\hline 11 & failed & failed & check & passed & 1 & passed & check & check & passed & passed & passed & & passed & \\
\hline 12 & failed & failed & failed & check & passed & 1 & passed & check & passed & passed & passed & & passed & \\
\hline 13 & check & check & passed & passed & passed & & passed & & passed & passed & passed & & passed & \\
\hline 14 & check & passed & passed & passed & I & passed & passed & & passed & passed & passed & & passed & \\
\hline 15 & check & check & passed & passed & passed & & passed & & passed & passed & passed & & passed & \\
\hline 16 & passed & passed & check & check & 1 & passed & passed & passed & passed & passed & passed & & passed & \\
\hline 17 & failed & failed & passed & passed & passed & & check & passed & passed & passed & passed & & passed & \\
\hline 18 & passed & failed & passed & passed & 1 & & passed & & passed & passed & passed & & passed & \\
\hline 19 & check & failed & failed & passed & 1 & & check & passed & check & passed & passed & & failed & check \\
\hline 20 & passed & check & passed & passed & passed & & failed & passed & passed & passed & passed & & passed & \\
\hline 21 & check & check & failed & check & I & & failed & failed & passed & passed & check & failed & passed & \\
\hline 22 & passed & check & check & check & passed & & failed & passed & passed & passed & passed & & passed & \\
\hline 23 & passed & check & passed & passed & passed & & passed & & passed & passed & passed & & passed & \\
\hline 24 & passed & passed & passed & passed & passed & & passed & & passed & passed & passed & & passed & \\
\hline 25 & passed & failed & passed & passed & I & & passed & passed & passed & passed & passed & & passed & \\
\hline 26 & check & check & passed & passed & passed & & check & passed & passed & passed & passed & & check & check \\
\hline 27 & check & passed & passed & passed & passed & & passed & & failed & 1 & I & & 1 & \\
\hline 28 & passed & passed & passed & passed & passed & & failed & failed & passed & passed & passed & & passed & \\
\hline 29 & check & check & passed & passed & passed & & passed & & passed & passed & passed & & passed & \\
\hline 30 & passed & passed & passed & passed & failed & check & check & passed & passed & passed & passed & & passed & \\
\hline 31 & failed & failed & passed & passed & 1 & & passed & & passed & passed & passed & & passed & \\
\hline 32 & passed & passed & passed & passed & passed & & passed & & passed & passed & passed & & passed & \\
\hline 33 & passed & passed & check & check & passed & I & check & passed & passed & passed & passed & & passed & \\
\hline 34 & check & check & passed & passed & 1 & & failed & passed & passed & passed & passed & & passed & \\
\hline 35 & check & failed & passed & passed & 1 & & failed & passed & passed & passed & passed & & passed & \\
\hline 36 & passed & passed & passed & passed & failed & failed & passed & & passed & passed & passed & & passed & \\
\hline 37 & check & passed & check & failed & passed & passed & failed & failed & passed & passed & passed & & check & check \\
\hline 38 & passed & passed & check & check & passed & passed & passed & & passed & passed & passed & & failed & failed \\
\hline 39 & check & check & passed & check & passed & & check & passed & passed & passed & passed & & passed & \\
\hline 40 & check & check & passed & passed & I & & failed & passed & passed & passed & passed & & passed & \\
\hline
\end{tabular}

From left to right the table gives results for DNA from FFPE tissues extracted with the classical method (FFPE DNA 1), extracted with the dedicated method (FFPE DNA 2) and DNA from cryo-preserved tissues (Cryo. DNA). "Passed" indicates a good conversion ( 0.0 to $5.0 \%$ of unconverted cytosines). "Check" indicates an uncertain conversion (5.0 to $7.0 \%$ of unconverted cytosines). "Failed" indicates a poor conversion (more than $7.0 \%$ of unconverted cytosines). Slash bar indicates there was no amplification. Results in bold emphasize controls of conversion giving "check" or "failed" results. 
had an invalid control of conversion, the other 38 DNAs (97.4\%) displayed satisfactory bisulfite conversion (DNA $\mathrm{n}^{\circ} 27$ was not amplified). The results were similar after the second assay. For the MGMT marker, in the first analysis, bisulfite conversion was not successful for five cases $\left(\mathrm{n}^{\circ} 7,19,26,37\right.$ and 38$)$ and DNA $n^{\circ} 27$ was not amplified. So $87.2 \%$ of cases showed valid controls of conversion. The same results were obtained in the second analysis.

\section{Comparison of the controls of conversion obtained for the three markers on the same DNA sample}

The results of the conversion controls were not always similar for a given DNA sample according to the marker used. FFPE DNAs $n^{\circ} 5,7,12,16,22,37$ and 38 showed good control of conversion with the MLH1 marker but an uncertain or failed control of conversion with the LINE-1 marker. Opposite results were observed for the two FFPE DNAs $\mathrm{n}^{\circ} 30$ and 36 . When amplified for the MGMT marker, FFPE DNAs $\mathrm{n}^{\circ} 5,12,16,22,33$ and 38 showed good control of conversion but an uncertain or failed control of conversion with the LINE-1 marker. Opposite results were observed for the two FFPE DNAs $n^{\circ} 28$ and 11. For cryo-preserved tissues, DNA n 21 displayed a valid control of conversion for $L I N E-1$ and MGMT markers and an uncertain or failed control of conversion when amplified for the $M L H 1$ marker. During MGMT amplification, five DNAs (cases $\mathrm{n}^{\circ} 7,19,26$, $37,38)$ showed uncertain or failed controls of conversion but valid controls of conversion for LINE-1 and MLH1 markers.

\section{Quantitative analysis of methylation levels for cryo- preserved and FFPE tissues (DNA extracted with the dedicated kit)}

The methylation levels of the 40 couples of DNA extracted from the same sample (cryo-preserved or FFPE) for the three markers are given in Table 3. Considering our previous results on the variability of the methylation level measure of DNA from cryo-preserved tissues for the LINE-1 marker (Table 1), we selected the minimal and maximal values of the methylation level for each CpG site. We calculated the difference between the two values to set up a threshold beyond which the difference in the methylation value was not due to the variability of measurements. The differences were $5.6 \%$ for CpG site 1, 4.1\% for CpG site 2 and $4.9 \%$ of methylation for $\mathrm{CpG}$ site 3. So we set an arbitrary threshold of $6.0 \%$ beyond which the difference was considered significant.

We chose not to show methylation levels of FFPE DNA extracted with the classical method because most of these DNAs displayed invalid conversion. Nevertheless we noticed that these levels were often higher than the levels of methylation of the same FFPE DNA extracted with the dedicated kit because $40.0 \%$ of the cases showed a significantly higher level of methylation (Additional file 2).

Eight couples $\left(\mathrm{n}^{\circ} 10,11,13,18,19,25,28\right.$ and 29) presented a difference in $L I N E-1$ methylation greater than $+6.0 \%$ or lower than $-6.0 \%$ between cryo-preserved and FFPE tissue. For these eight cases, the bisulfite conversion control was successful for all cryo-preserved and FFPE DNAs. Among these eight couples, the level of methylation in five cases was higher for DNA extracted from cryo-preserved tissue than from FFPE tissue and lower in three cases. For the MLH1 marker, the differences could be established on 29 couples of DNA. Four couples $(1,9,13$ and 17) displayed a difference in the MLH1 methylation level greater than $+6.0 \%$ or lower than $-6.0 \%$ with no problem of bisulfite conversion. Among these four couples, three had a level of $M L H 1$ methylation that was higher in the DNA extracted from cryo-preserved tissue. With the MGMT marker, 15 couples $\left(\mathrm{n}^{\circ} 3,4,6,7,10,11,17,19,20,24,25,26,28,32\right.$ and 37) of DNA presented a difference in the level of MGMT methylation greater than $+6.0 \%$ or lower than $-6.0 \%$. Seven of these couples (cases $n^{\circ}$ $3,7,11,19,26,28,37)$ showed a problem of bisulfite conversion for one of the two samples. Among the remaining eight couples, the methylation level was higher for DNA extracted from FFPE tissue in six cases $\left(n^{\circ} 6,10,17,20\right.$, 24 and 32 ) and lower for two cases ( $n^{\circ} 4$ and 25). By combining the analyses of the methylation levels of the three markers (Table 3), no couples of frozen/FFPE DNA presented a difference in the methylation level for the three markers at the same time. Five couples of DNA ( ${ }^{\circ} 10,11,19,25$ and 28) displayed a difference in methylation levels for both LINE-1 and MGMT markers, one couple $\left(\mathrm{n}^{\circ} 13\right)$ for $L I N E-1$ and $M L H 1$ markers and one couple $\left(\mathrm{n}^{\circ} 17\right)$ for $M L H 1$ and MGMT. Examples of pyrograms for couples showing differences in methylation levels are presented in Figure 2.

\section{Synthetic analysis of bisulfite conversion quality and} methylation levels for cryo-preserved and FFPE tissues for the three markers (Table 4)

For the Line 1 marker, 29 couples had successful bisulfite conversion. Among these, eight (27.6\%) displayed a significant difference in the methylation levels while 21 couples showed no difference in the methylation levels between cryo-preserved and FFPE samples. For the MLH1 marker, 26 out of 28 couples for which DNA could be amplified showed satisfactory control of bisulfite conversion. Among these, four (15.4\%) displayed a significant difference in the methylation levels while 22 couples showed no difference in the methylation levels between cryo-preserved and FFPE samples. For the 
Table 3 Differences in methylation levels for each couple of frozen/FFPE DNA according to the three markers

\begin{tabular}{|c|c|c|c|c|c|c|c|c|c|}
\hline \multirow[t]{2}{*}{ Identity } & \multicolumn{2}{|c|}{$\begin{array}{l}\text { Levels of LINE-1 } \\
\text { methylation } \\
\text { (\% of methylation) }\end{array}$} & \multirow[t]{2}{*}{$\begin{array}{l}\text { Difference } \\
\quad \text { of } \\
\text { methylation }\end{array}$} & \multicolumn{2}{|c|}{$\begin{array}{l}\text { Levels of } M L H 1 \\
\text { methylation } \\
\text { (\% of methylation) }\end{array}$} & \multirow[t]{2}{*}{$\begin{array}{l}\text { Difference } \\
\quad \text { of } \\
\text { methylation }\end{array}$} & \multicolumn{2}{|c|}{$\begin{array}{l}\text { Levels of } M G M T \\
\text { methylation } \\
\text { (\% of methylation) }\end{array}$} & \multirow[t]{2}{*}{$\begin{array}{l}\text { Difference } \\
\text { of } \\
\text { methylation }\end{array}$} \\
\hline & $\begin{array}{l}\text { DNA from cryo- } \\
\text { preserved } \\
\text { tissues }\end{array}$ & $\begin{array}{l}\text { DNA from } \\
\text { FFPE } \\
\text { tissues }\end{array}$ & & $\begin{array}{l}\text { DNA from } \\
\text { cryo-preserved } \\
\text { tissues }\end{array}$ & $\begin{array}{l}\text { DNA from } \\
\text { FFPE } \\
\text { tissues }\end{array}$ & & $\begin{array}{l}\text { DNA from } \\
\text { cryo-preserved } \\
\text { tissues }\end{array}$ & $\begin{array}{l}\text { DNA from } \\
\text { FFPE } \\
\text { tissues }\end{array}$ & \\
\hline 1 & 62.0 & 66.3 & -4.3 & 51.5 & 57.7 & -6.2 & 1.7 & 4.3 & -2.6 \\
\hline 2 & 66.1 & 67.2 & -1.1 & 4.8 & - & - & 2.0 & 6.0 & -4.0 \\
\hline 3 & 64.6 & $64.8+$ & -0.2 & 47.6 & - & - & 57.5 & $71.9 \neq$ & -14.3 \\
\hline 4 & 57.2 & 52.3 & 5.0 & 6.8 & - & - & 55.8 & 49.3 & 6.5 \\
\hline 5 & 61.6 & $64.2+$ & -2.6 & 5.3 & $1,7^{*}$ & 3.6 & 1.9 & 6.2 & -4.2 \\
\hline 6 & 47.5 & 49.2 & -1.7 & 2.9 & 3.9 & -1.0 & 38.6 & 46.1 & -7.5 \\
\hline 7 & 59.4 & $56.6+$ & 2.9 & 5.1 & 1.9 & 3.3 & $59.4+$ & 43.4 & 16.0 \\
\hline 8 & 47.9 & 46.5 & 1.3 & 3.4 & $1,5^{*}$ & 1.9 & 1.7 & 5.7 & -4.0 \\
\hline 9 & 64.5 & 61.2 & 3.3 & 33.1 & 13.3 & 19.7 & 14.7 & 18.2 & -3.5 \\
\hline 10 & 61.4 & 54.1 & 7.3 & 5.6 & 1.4 & 4.2 & 19.2 & 32.3 & -13.1 \\
\hline 11 & 60.5 & 50.1 & 10.4 & 3.9 & $3,4^{*}$ & 0.6 & 19.8 & $35.6+$ & -15.8 \\
\hline 12 & 60.2 & $62.2+$ & -2.0 & 4.1 & 2.8 & 1.3 & 2.0 & 5.1 & -3.2 \\
\hline 13 & 45.1 & 62.5 & -17.5 & 45.2 & 2.6 & 42.7 & 2.1 & 4.3 & -2.2 \\
\hline 14 & 57.0 & 60.8 & -3.8 & 3.0 & $7,1^{*}$ & -4.1 & 1.4 & 1.9 & -0.5 \\
\hline 15 & 59.2 & 63.2 & -4.0 & 2.5 & 5.6 & -3.1 & 1.4 & 4.9 & -3.5 \\
\hline 16 & 52.8 & $47.5+$ & 5.2 & 2.6 & $3,3^{*}$ & -0.7 & 1.6 & 5.9 & -4.3 \\
\hline 17 & 61.0 & 55.6 & 5.3 & 77.7 & 4.6 & 73.1 & 1.7 & 30.2 & -28.5 \\
\hline 18 & 59.6 & 53.5 & 6.2 & 4.8 & - & - & 28.9 & 25.3 & 3.6 \\
\hline 19 & 43.3 & 54.4 & -11.2 & 2.2 & & - & $73.9+$ & 62.2 & 11.7 \\
\hline 20 & 61.7 & 57.6 & 4.1 & 4.6 & 3.5 & 1.1 & 15.7 & 25.2 & -9.5 \\
\hline 21 & 65.2 & $66.1+$ & -1.0 & $6.1+$ & - & - & 2.2 & $7.0 \neq$ & -4.8 \\
\hline 22 & 50.9 & $49.1+$ & 1.8 & 3.5 & 3.3 & 0.3 & 1.9 & 6.8 & -5.0 \\
\hline 23 & 61.6 & 59.4 & 2.2 & 3.5 & 2.1 & 1.4 & 1.6 & 4.6 & -2.9 \\
\hline 24 & 59.3 & 58.0 & 1.4 & 2.4 & 3.5 & -1.1 & 20.8 & 32.4 & -11.7 \\
\hline 25 & 61.7 & 71.0 & -9.3 & 4.1 & - & - & 31.7 & $1,9^{*}$ & 29.8 \\
\hline 26 & 56.7 & 52.2 & 4.4 & 2.4 & 0.9 & 1.5 & $52.8+$ & 20.4 & 32.4 \\
\hline 27 & $45.5 \neq$ & 44.3 & 1.3 & - & 5.9 & - & - & 4.2 & - \\
\hline 28 & 65.3 & 57.4 & 7.9 & 6.6 & $2,7^{*}$ & 3.9 & 41.8 & $25.2 \neq$ & 16.6 \\
\hline 29 & 64.0 & 57.3 & 6.7 & 3.4 & $2,4^{*}$ & 1.0 & 2.2 & 3.3 & -1.1 \\
\hline 30 & 56.4 & 56.7 & -0.3 & 11.5 & 7.3 & 4.3 & 3.9 & 5.3 & -1.5 \\
\hline 31 & 62.0 & 62.9 & -1.0 & 32.8 & - & - & 5.7 & 5.3 & 0.4 \\
\hline 32 & 67.2 & 62.0 & 5.2 & 3.9 & 5.2 & -1.2 & 19.0 & 51.8 & -32.8 \\
\hline 33 & 60.2 & $62.4+$ & -2.3 & 4.0 & 3.9 & 0.1 & 2.0 & 7.9 & -5.9 \\
\hline 34 & 62.0 & 61.7 & 0.3 & 3.6 & - & - & 2.2 & 5.9 & -3.7 \\
\hline 35 & 56.9 & 53.3 & 3.5 & 3.8 & - & - & 2.1 & 5.6 & -3.5 \\
\hline 36 & 40.5 & 41.1 & -0.6 & 3.0 & 3.6 & -0.6 & 1.7 & 5.2 & -3.5 \\
\hline 37 & 59.0 & $62.1+$ & -3.0 & 2.7 & 3.3 & -0.6 & $88.3+$ & $73.1 \neq$ & 15.1 \\
\hline 38 & 62.3 & $58.5+$ & 3.8 & 4.3 & 1.5 & 2.8 & 68.2 & 71.7 & -3.5 \\
\hline 39 & 50.4 & 53.2 & -2.9 & 4.8 & 4.5 & 0.3 & 1.9 & 4.6 & -2.7 \\
\hline 40 & 36.2 & 34.7 & 1.5 & 3.1 & - & - & 2.0 & 5.9 & -4.0 \\
\hline
\end{tabular}

Values of methylation levels correspond to the average level of methylation of the three CpG sites evaluated for LINE-1 and the five CpG sites evaluated for MLH1 and MGMT. Data were collected using the pyrogram which had the best internal control of conversion.

*: indicates an uncertain result due to the low intensity of the pyrogram. t: indicates an uncertain conversion (5.0 to $7.0 \%$ of unconverted cytosines). $\neq:$ indicates a poor conversion (more than $7.0 \%$ of unconverted cytosines). Numbers in bold indicate a difference of methylation level outside the $-6.0 /+6.0 \%$ interval. Differences in methylation levels were calculated by subtracting the level of methylation of the cryo-preserved sample from the level of methylation of the FFPE sample. 


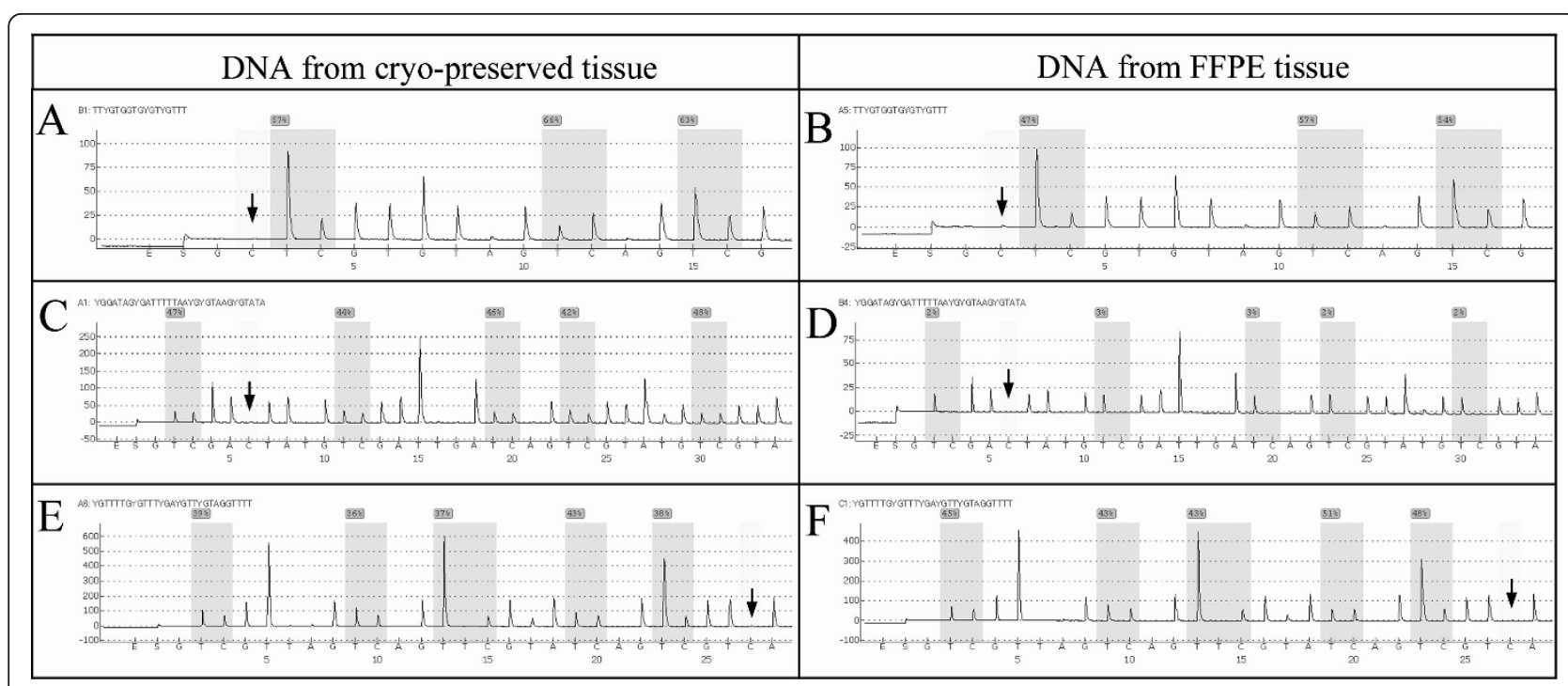

Figure 2 Pyrograms of the LINE-1, MLH1 and MGMT methylation markers for different couples of frozen/FFPE DNA. Pyrograms of LINE-1 marker are those obtained for couple $n^{\circ} 10(\mathbf{A}$ and $\mathbf{B})$ and for MLH1 and MGMT markers those for couples $n^{\circ} 13$ (C and $\left.\mathbf{D}\right)$ and $n^{\circ} 6(\mathbf{E}$ and $\mathbf{F})$ respectively. Arrows indicate positions of internal controls of conversion, demonstrating no residual cytosines at the non-CpG sites. Gray areas indicate polymorphisms, between T/C, generated by bisulfite treatment. Level of methylation for a given CpG dinucleotide is reported above it (gray square).

MGMT marker, 31 couples had successful bisulfite conversion. Among these, eight (25.8\%) displayed a significant difference in the methylation levels while 23 couples showed no difference in the methylation levels between cryo-preserved and FFPE samples. All things considered, five couples $\left(\mathrm{n}^{\circ} 8,14,15,23,39\right)$ displayed both satisfactory control of conversion and no difference in the methylation levels for the three markers.

\section{Discussion}

Epigenetic silencing of genes, mostly mediated by aberrant DNA methylation, is a mechanism of gene inactivation in patients with colorectal cancer [2]. Among the loci that can undergo aberrant methylation in colorectal cancer, one subgroup appears to become aberrantly methylated as a specific group $[2,4]$, a phenomenon called the $\mathrm{CpG}$ island methylator phenotype (CIMP). Results in the literature on CIMP status as a prognostic factor for colon cancers remain controversial. These conflicting results could result from differences in patient cohorts, samples (the use of frozen or FFPE tissues), analytical techniques, methylation markers (different genes or the same genes but screened at different sites), primer sequences and criteria for CIMP. In our study, we chose to focus on the impact of the preanalytical phase. A variety of assays to measure DNA methylation have been developed for FFPE and frozen tissues many of which rely on the bisulfite conversion of unmethylated cytosines from tumour tissue into uracils. However, the efficacy of sodium bisulfite treatment and the measurement of methylation levels in FFPE samples in one hand and in frozen samples on the other hand have never really been compared and evaluated.

In this study, we assessed the quality of bisulfite conversion as well as methylation levels for LINE-1, MLH1 and MGMT markers by pyrosequencing from 40 pairs of FFPE and frozen samples. Pyrosequencing is particularly useful because it provides resolution at the individual nucleotide level and includes a conversion control for each analysis. Assays targeting CpG islands of LINE1, MLH1 and MGMT were chosen since aberrant methylation of this retro-transposon and these genes is implicated in colon cancers. LINE-1 (long interspersed nucleotide element-1) is a retro-transposable element of

Table 4 Synthetic view of the bisulfite conversion quality and the equivalence of methylation levels

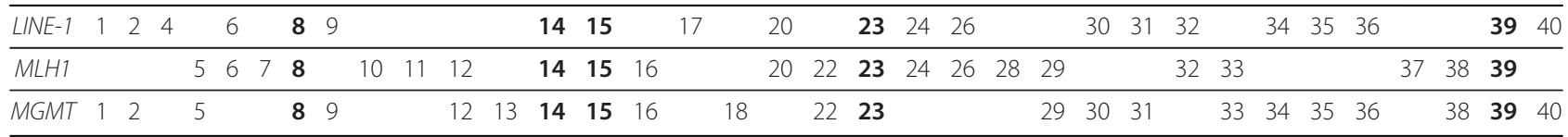

In each line (according to the corresponding marker) the identities of couples of frozen/FFPE DNAs (designated as couple 1 through couple 40 ) displaying both successful controls of bisulfite conversion and no differences in methylation levels are listed. Numbers in bold indicates couples displaying both a successful control of bisulfite conversion and no differences in methylation levels for the three markers. 
DNA that is present in $15 \%$ of the human genome. It is a surrogate marker of genome-wide DNA methylation $[18,22,23]$. It is frequently hypomethylated in human cancers $[24,25]$. In contrast, the two genes $M L H 1$ (the human homolog of the E. coli DNA mismatch repair gene mutL) and MGMT (O6-Methylguanine DNA methyltransferase) are hypermethylated in a number of different cancers, including colorectal cancer [26-28].

We first demonstrated that bisulfite-to-bisulfite standard deviations of methylation levels (mean 1.3\%) and $\mathrm{PCR} /$ pyrosequencing run-to-run standard deviations of methylation levels (mean $0.9 \%$ ) were low and acceptable. We chose to carry out the assays on frozen tissues to avoid the potential impact of DNA degradation from FFPE tissues. The results obtained for LINE - 1 can be extrapolated to any other validated methylated markers. These assays allowed us to consider that our process of bisulfite conversion and PCR/pyrosequencing is reproducible and to establish an arbitrary threshold of $6.0 \%$ beyond which the difference in methylation value was not due to variability in the measurements.

To assess the quality of sodium bisulfite conversion and the subsequent PCR/pyrosequencing assays, using the LINE-1 marker, we evaluated conversion of 40 FFPE DNAs extracted using a routine method. The results were neither satisfactory nor reliable since only 15 cases showed valid control of conversion after the first analysis and 22 cases (55.0\%) after combining the two analyses. We consequently decided to use a dedicated kit to re-extract DNA from FFPE tissues and to extend the assays to the two other markers. All the samples were evaluated using the LINE-1 and MGMT markers: only 27 cases (67.5\%) showed good control of bisulfite conversion with the LINE-1 marker and 22 cases $(55.0 \%)$ with the MGMT marker in the first assay. In contrast, with the $M L H 1$ marker, in 11 cases amplification failed, possibly because of the length of the MLH1 amplicon (181 pb). Among the 29 amplifiable samples, 23 (57.5\% of the 40 cases) showed valid control of conversion in the first analysis. Furthermore, the results were not the same in the second analysis. It is thus clear that the extraction phase should not be conducted using a routine process and must comprise a supplementary step of heating to $90^{\circ} \mathrm{C}$ for $1 \mathrm{~h}$ to improve bisulfite conversion. Even with this dedicated method, the rate of satisfactorily converted samples was much lower for FFPE than for frozen samples. In contrast, the proportion of satisfactorily converted samples in the first assay on frozen samples was $95.0 \%$ with $L I N E-1,97.4 \%$ with $M L H 1$ and $87.2 \%$ with MGMT. The results were reliable and similar after the second analysis. Furthermore, we did not encounter the problem of failed amplification (due to amplicon length) found with the $M L H 1$ marker on FFPE tissues. DNA derived from FFPE is an extremely valuable source of material for retrospective studies, but is often highly degraded. When PCR-based methods are used to study DNA methylation changes, it is necessary to modify the DNA with sodium bisulfite to preserve the DNA methylation information of the original template, and this treatment may further damage the DNA. Fragmentation of FFPE DNA is a real drawback, particularly in DNA methylation studies based on methylationindependent PCR: the design of the primers must be conducted with the highest score, primers have to hybridize out of the $\mathrm{CpG}$ islands, and amplicon length has to be limited. It is highly required to respect these constraints when using pyrosequencing which is the only technique allowing a real quantification of methylation.

In our study, FFPE DNAs ( ${ }^{\circ} 30$ and 36 ), showed invalid control of conversion with $M L H 1$ and good control with $L I N E-1$ and MGMT. In addition, none of the FFPE DNAs showing invalid control of bisulfite conversion with LINE1 (10 cases) showed invalid conversion with $M L H 1$, while in three cases (cases $n^{\circ} 3,21$ and 37) the results coincided with those of MGMT. As the efficacy of bisulfite conversion appeared to be heterogeneous all along the DNA and variable according to the analysed marker, we think that percentages of bisulfite conversion differ from one cytosine to another due to residual cross-linkings (even with the $90^{\circ} \mathrm{C}$ heating step) leading to non-reproducible results on FFPE samples with the three markers.

Thus, even when extraction methods dedicated to FFPE tissue were used, problems occurred with bisulfite conversion. The importance of successful bisulfite conversion was underlined by a panel of experts who reported that incomplete conversion of DNA was the major cause of false-positive results in methylation analysis [29]. We chose not to evaluate the results of the methylation levels obtained from FFPE DNA extracted using the classical method because the number of unsatisfactory conversions was too high, but it was clear that poor bisulfite conversion led to overestimation of methylation (Additional file 3: Figure 1). In order to compare the methylation levels of cryo-preserved and FFPE samples (DNA extraction with the dedicated kit), we established an arbitrary threshold (6.0\%) beyond which differences in the methylation value were not due to variability in the measurements. We demonstrated that with $L I N E-1$ eight pairs (27.6\%), with $M L H 1$, four pairs (15.4\%), and with MGMT, eight pairs (25.8\%) displayed significant differences in the methylation level. These deviations in methylation levels between matched FFPE and cryo-preserved samples cannot be due to differences in tumour cellularity as we checked that they were slight. In a previous work (data not published) we evaluated the variations in methylation levels induced by variations in tumor cellularity using the LINE-1 marker, and we observed a maximal deviation of $3.6 \%$ of 
methylation for a cellularity difference of around 20\% from the same sample. This argument is supported by the study published by Irahara et al. [30] who compared average methylation values for LINE-1 in macrodissected colon cancers with those for matched Laser Capture Microdissection specimens providing a pure collection of tumor cells. They found no substantial effects of contaminating normal cells on LINE-1 methylation.

We found no pairs that showed a difference in methylation level with all three markers and we were unable to establish a trend for the differences in methylation levels in FFPE versus frozen samples. Clearly these deviations in methylation levels differentially impact according to the analyzed marker. For MLH1 and MGMT markers, a sample is considered as methylated whatever it displayed $20 \%$ or $50 \%$ of methylation. But for the LINE-1 marker, several subgroups of methylation can be distinguished and variations of methylation shown here can be critical for the creation of these subgroups.

Our study is the first to compare the results obtained for DNA extracted from FFPE and frozen tissues to assess the feasibility of DNA methylation analysis using pyrosequencing. Some authors maintain that sodium bisulfite treatment is sufficiently precise and shows good reproducibility on FFPE samples leading to reliable assessment of methylation levels [19,31]. Nevertheless, these authors did not perform comparative studies with frozen material and used techniques such as Methylight (real time PCR), SMART-MSP (Sensitive Melting Analysis after Real Time PCR - methylation-specific polymerase chain reaction), MS-HRM (Methylation SensitiveHigh Resolution Melting), without a built-in measure to check the completeness of bisulfite conversion (conversion control). Furthermore, to confirm that FFPE tissue can be effectively used for high-throughput DNA methylation analysis, Ogino et al. [31] used the alternative method of protein expression by immunohistochemistry, which is a surrogate indicator of DNA methylation.

Looking ahead, there is another source of variability that should be investigated: bisulfite conversion methods which vary according to laboratories. This point reinforces the need for standardization in this domain.

\section{Conclusions}

In conclusion, we demonstrated that the use of FFPE tissues induces unsatisfactory and non-reproducible bisulfite conversion leading to unreliable results for methylation levels. In contrast, frozen samples give reproducible results for sodium bisulfite conversion and subsequent pyrosequencing assays have acceptable precision using a single analysis. There is clearly a need to standardize the entire process of DNA methylation analysis from the tissue preservation method to the technology used to quantify methylation. Our results indicate that using FFPE collections to evaluate the prognosis of CIMP colon cancers by bisulfite methods partly contributes to the discrepant data observed in this field. In light of these results, we strongly recommend the use of DNA from cryo-preserved tissue when performing bisulfite conversion to study DNA methylation.

\section{Additional material}

Additional file 1: This file is in Microsoft Word $\mathbf{9 7 - 2 0 0 3}$ format. It
includes a detailed description of the protocol for the extraction method
dedicated to FFPE tissue and of the protocol for the DNA bisulfite
conversion
Additional file 2: This file is in Microsoft Word $\mathbf{9 7 - 2 0 0 3}$ format. This
file contains the LINE-1 primer sequences (Supplementary table 2), the
dispensation orders of nucleotides for the three markers (Supplementary
table 2) and data on the LINE-1 methylation levels obtained from FFPE
DNA extracted by the classical method (Supplementary table 3).
Additional file 3: This file is in JPEG format. This figure is entitled:
Pyrograms of the LINE-1 methylation marker for FFPE DNA $n^{\circ} 2$ extracted
with the classical method. It groups the two pyrograms obtained from
the two LINE-1 analyses of the FFPE DNA $n^{\circ} 2$ extracted with the classical
method.

\section{Abbreviations}

BAX: BCl2-associated $\times$ protein; CIMP: CpG island methylator phenotype; CpG: Cytosine-phosphate-Guanine; DNA: deoxyribonucleic acid; FFPE: formalinfixed paraffin embedded; HES: Hematein-Eosin-Safran; IGFR: insulin-like growth factor receptor; LINE-1: Long Interspersed nucleotide element 1; MGMT: O6-Methylguanine DNA methyltransferase; MLH1: human MutL homolog 1; MS-HRM: methylation sensitive-high resolution melting; MSH3: human MutS homolog 3; MSP: methylation-specific polymerase chain reaction; MSS: microsatellite stable; $P C R$ : polymerase chain reaction; RNase: ribonuclease; dNTP: deoxy-nucleotide triphosphate; SD: standard deviation; SMART-MSP: sensitive melting analysis after real time PCR - methylationspecific polymerase chain reaction; TGF $\beta R / l$ : transforming growth factor-beta receptor, type $\|$

\section{Acknowledgements}

We thank the Biological Resource Centre of Dijon (Ferdinand Cabanne) and we thank Philip Bastable of the Centre of Research at Dijon CHU for reviewing the English.

\section{Author details}

'Institut National de la Sante et de la Recherche Médicale, Université de Bourgogne, U866 Dijon, France. ${ }^{2}$ Service de Pathologie, CHU, Dijon, France. ${ }^{3}$ Registre Bourguignon des Cancers Digestifs, CHU, Dijon, France. ${ }^{4}$ Centre de ressources biologiques Ferdinand Cabanne, Dijon, France.

\section{Authors' contributions}

$B T, C C, C L$ and FP instigated the study. EC and LM participated in the study design. BT carried out the DNA extractions and the pyrosequencing experiments. BT and FP drafted the manuscript. CC, CL and JF were involved in revising it critically. All authors read and approved the final manuscript.

\section{Competing interests}

The authors declare that they have no competing interests.

Received: 30 June 2011 Accepted: 13 January 2012

Published: 13 January 2012

\section{References}

1. Esteller M: Epigenetics in cancer. N Engl J Med 2008, 358:1148-1159.

2. Issa JP: CpG island methylator phenotype in cancer. Nat Rev Cancer 2004, 4:988-993. 
3. Wong JJ, Hawkins NJ, Ward RL: Colorectal cancer: a model for epigenetic tumorigenesis. Gut 2007, 56:140-148.

4. Toyota M, Ahuja N, Ohe-Toyota M, Herman JG, Baylin SB, Issa JP: CpG island methylator phenotype in colorectal cancer. Proc Natl Acad Sci USA 1999, 96:8681-8686.

5. Barault L, Charon-Barra C, Jooste V, de la Vega MF, Martin L, Roignot $P$, Rat P, Bouvier AM, Laurent-Puig P, Faivre J, et al: Hypermethylator phenotype in sporadic colon cancer: study on a population-based series of 582 cases. Cancer Res 2008, 68:8541-8546.

6. Ferracin M, Gafa R, Miotto E, Veronese A, Pultrone C, Sabbioni S, Lanza G, Negrini M: The methylator phenotype in microsatellite stable colorectal cancers is characterized by a distinct gene expression profile. J Pathol 2008, 214:594-602.

7. Lee S, Cho NY, Choi M, Yoo EJ, Kim JH, Kang GH: Clinicopathological features of $\mathrm{CpG}$ island methylator phenotype-positive colorectal cancer and its adverse prognosis in relation to KRAS/BRAF mutation. Pathol Int 2008, 58:104-113.

8. Ogino S, Meyerhardt JA, Kawasaki T, Clark JW, Ryan DP, Kulke MH, Enzinger PC, Wolpin BM, Loda M, Fuchs CS: CpG island methylation, response to combination chemotherapy, and patient survival in advanced microsatellite stable colorectal carcinoma. Virchows Arch 2007, 450:529-537.

9. Ogino S, Nosho K, Kirkner GJ, Kawasaki T, Meyerhardt JA, Loda M, Giovannucci EL, Fuchs CS: CpG island methylator phenotype, microsatellite instability, BRAF mutation and clinical outcome in colon cancer. Gut 2009, 58:90-96

10. Samowitz WS, Sweeney C, Herrick J, Albertsen H, Levin TR, Murtaugh MA, Wolff RK, Slattery ML: Poor survival associated with the BRAF V600E mutation in microsatellite-stable colon cancers. Cancer Res 2005, 65:6063-6069.

11. Shen L, Catalano PJ, Benson AB, O'Dwyer P, Hamilton SR, Issa JP: Association between DNA methylation and shortened survival in patients with advanced colorectal cancer treated with 5-fluorouracil based chemotherapy. Clin Cancer Res 2007, 13:6093-6098.

12. Van Rijnsoever M, Elsaleh H, Joseph D, McCaul K, lacopetta B: CpG island methylator phenotype is an independent predictor of survival benefit from 5-fluorouracil in stage III colorectal cancer. Clin Cancer Res 2003, 9:2898-2903.

13. Ward RL, Cheong K, Ku SL, Meagher A, O'Connor T, Hawkins NJ: Adverse prognostic effect of methylation in colorectal cancer is reversed by microsatellite instability. J Clin Oncol 2003, 21:3729-3736.

14. Frommer M, McDonald LE, Millar DS, Collis CM, Watt F, Grigg GW, Molloy PL, Paul CL: A genomic sequencing protocol that yields a positive display of 5-methylcytosine residues in individual DNA strands. Proc Natl Acad Sci USA 1992, 89:1827-1831.

15. Clark SJ, Harrison J, Paul CL, Frommer M: High sensitivity mapping of methylated cytosines. Nucleic Acids Res 1994, 22:2990-2997.

16. Laird PW: Principles and challenges of genomewide DNA methylation analysis. Nat Rev Genet 11:191-203.

17. Uhlmann K, Brinckmann A, Toliat MR, Ritter H, Nurnberg P: Evaluation of a potential epigenetic biomarker by quantitative methyl-single nucleotide polymorphism analysis. Electrophoresis 2002, 23:4072-4079.

18. Yang AS, Estecio MR, Doshi K, Kondo Y, Tajara EH, Issa JP: A simple method for estimating global DNA methylation using bisulfite PCR of repetitive DNA elements. Nucleic Acids Res 2004, 32:e38.

19. Kristensen LS, Wojdacz TK, Thestrup BB, Wiuf C, Hager H, Hansen LL: Quality assessment of DNA derived from up to 30 years old formalin fixed paraffin embedded (FFPE) tissue for PCR-based methylation analysis using SMART-MSP and MS-HRM. BMC Cancer 2009, 9:453.

20. Dietmaier W, Wallinger S, Bocker T, Kullmann F, Fishel R, Ruschoff J: Diagnostic microsatellite instability: definition and correlation with mismatch repair protein expression. Cancer Res 1997, 57:4749-4756.

21. Suraweera N, Duval A, Reperant M, Vaury C, Furlan D, Leroy K, Seruca R, lacopetta B, Hamelin R: Evaluation of tumor microsatellite instability using five quasimonomorphic mononucleotide repeats and pentaplex PCR. Gastroenterology 2002, 123:1804-1811.

22. Yang AS, Doshi KD, Choi SW, Mason JB, Mannari RK, Gharybian V, Luna R, Rashid A, Shen L, Estecio MR, et al: DNA methylation changes after 5-aza2'-deoxycytidine therapy in patients with leukemia. Cancer Res 2006, 66:5495-5503.
23. Weisenberger DJ, Campan M, Long TI, Kim M, Woods C, Fiala E, Ehrlich M, Laird PW: Analysis of repetitive element DNA methylation by MethyLight. Nucleic Acids Res 2005, 33:6823-6836.

24. Chalitchagorn K, Shuangshoti S, Hourpai N, Kongruttanachok N, Tangkijvanich P, Thong-ngam D, Voravud N, Sriuranpong V, Mutirangura A: Distinctive pattern of LINE-1 methylation level in normal tissues and the association with carcinogenesis. Oncogene 2004, 23:8841-8846.

25. Phokaew C, Kowudtitham S, Subbalekha K, Shuangshoti S, Mutirangura A: LINE-1 methylation patterns of different loci in normal and cancerous cells. Nucleic Acids Res 2008, 36:5704-5712.

26. Toyota M, Suzuki H: Epigenetic drivers of genetic alterations. Adv Genet 70:309-323.

27. Mirchev MB, Kahl P, Friedrichs N, Kotzev IA, Buettner R: DNA methylation in patients with colorectal cancer-correlation with some clinical and morphological features and with local tumour invasion. Folia Med (Plovdiv) 52:22-30

28. Shima K, Morikawa T, Baba Y, Nosho K, Suzuki M, Yamauchi M, Hayashi M, Giovannucci E, Fuchs CS, Ogino S: MGMT promoter methylation, loss of expression and prognosis in 855 colorectal cancers. Cancer Causes Control 22:301-309.

29. Kagan J, Srivastava S, Barker PE, Belinsky SA, Cairns P: Towards clinical application of methylated DNA sequences as cancer biomarkers: a joint NCl's EDRN and NIST workshop on standards, methods, assays, reagents and tools. Cancer Res 2007, 67:4545-4549.

30. Irahara N, Nosho K, Baba Y, Shima K, Lindeman NI, Hazra A, Schernhammer ES, Hunter DJ, Fuchs CS, Ogino S: Precision of pyrosequencing assay to measure LINE-1 methylation in colon cancer, normal colonic mucosa, and peripheral blood cells. J Mol Diagn 12:177-183.

31. Ogino S, Kawasaki T, Brahmandam M, Cantor M, Kirkner GJ, Spiegelman D, Makrigiorgos GM, Weisenberger DJ, Laird PW, Loda M, Fuchs CS: Precision and performance characteristics of bisulfite conversion and real-time PCR (MethyLight) for quantitative DNA methylation analysis. J Mol Diagn 2006, 8:209-217.

Pre-publication history

The pre-publication history for this paper can be accessed here: http://www.biomedcentral.com/1471-2407/12/12/prepub

doi:10.1186/1471-2407-12-12

Cite this article as: Tournier et al: Why do results conflict regarding the prognostic value of the methylation status in colon cancers? the role of the preservation method. BMC Cancer 2012 12:12.

\section{Submit your next manuscript to BioMed Central and take full advantage of:}

- Convenient online submission

- Thorough peer review

- No space constraints or color figure charges

- Immediate publication on acceptance

- Inclusion in PubMed, CAS, Scopus and Google Scholar

- Research which is freely available for redistribution

Submit your manuscript at www.biomedcentral.com/submit
C Biomed Central 